University of Nebraska - Lincoln

DigitalCommons@University of Nebraska - Lincoln

$9-24-2004$

\title{
Production of a keV X-Ray Beam from Synchrotron Radiation in Relativistic Laser-Plasma Interaction
}

\author{
Antoine Rousse \\ Laboratoire d'Optique Appliquée, ENSTA, CNRS UMR7639, Ecole Polytechnique, Chemin de la Hunière, \\ France \\ Kim Ta Phuoc \\ Laboratoire d'Optique Appliquée, ENSTA, CNRS UMR7639, Ecole Polytechnique, Chemin de la Hunière, \\ France \\ Rahul Shah \\ University of Michigan, Ann Arbor \\ Alexander Pukhov \\ Insitut fuer Theoretische Physik I, Heinrich-Heine-Universitaet, Duesseldorf,Germany \\ Eric Lefebvre \\ Département de Physique Théorique et Appliquée, CEA/DAM Ile- de-France, France \\ See next page for additional authors \\ Follow this and additional works at: https://digitalcommons.unl.edu/physicsumstadter \\ Part of the Physics Commons
}

Rousse, Antoine; Phuoc, Kim Ta; Shah, Rahul; Pukhov, Alexander; Lefebvre, Eric; Malka, Victor; Kiselev, Sergey; Burgy, Frederic; Rousseau, Jean-Philippe; Umstadter, Donald P.; and Hulin, Daniele, "Production of a keV X-Ray Beam from Synchrotron Radiation in Relativistic Laser-Plasma Interaction" (2004). Donald Umstadter Publications. 18.

https://digitalcommons.unl.edu/physicsumstadter/18

This Article is brought to you for free and open access by the Research Papers in Physics and Astronomy at DigitalCommons@University of Nebraska - Lincoln. It has been accepted for inclusion in Donald Umstadter Publications by an authorized administrator of DigitalCommons@University of Nebraska - Lincoln. 


\section{Authors}

Antoine Rousse, Kim Ta Phuoc, Rahul Shah, Alexander Pukhov, Eric Lefebvre, Victor Malka, Sergey Kiselev, Frederic Burgy, Jean-Philippe Rousseau, Donald P. Umstadter, and Daniele Hulin 


\title{
Production of a keV X-Ray Beam from Synchrotron Radiation in Relativistic Laser-Plasma Interaction
}

\author{
Antoine Rousse, ${ }^{1}$ Kim Ta Phuoc,,${ }^{1,2 * *}$ Rahul Shah,${ }^{2}$ Alexander Pukhov, ${ }^{3}$ Eric Lefebvre, ${ }^{4}$ Victor Malka,,${ }^{1}$ Sergey Kiselev, ${ }^{3}$ \\ Fréderic Burgy, ${ }^{1}$ Jean-Philippe Rousseau, ${ }^{1}$ Donald Umstadter, ${ }^{2}$ and Daniéle Hulin ${ }^{1}$ \\ ${ }^{1}$ Laboratoire d'Optique Appliquée, ENSTA, CNRS UMR7639, Ecole Polytechnique, \\ Chemin de la Hunière, 91761 Palaiseau, France. \\ ${ }^{2}$ Center for Ultrafast Optical Science, University of Michigan, 2200 Bonisteel Blvd, Ann Arbor, Michigan, 48109, USA. \\ ${ }^{3}$ Insitut fuer Theoretische Physik I, Heinrich-Heine-Universitaet, Duesseldorf, 40225 Duesseldorf, Germany. \\ ${ }^{4}$ Département de Physique Théorique et Appliquée, CEA/DAM Ile-de-France, BP 12, 91680 Bruyéres-le-Châtel, France
}

(Received 22 January 2004; published 23 September 2004)

\begin{abstract}
We demonstrate that a beam of $\mathrm{x}$-ray radiation can be generated by simply focusing a single highintensity laser pulse into a gas jet. A millimeter-scale laser-produced plasma creates, accelerates, and wiggles an ultrashort and relativistic electron bunch. As they propagate in the ion channel produced in the wake of the laser pulse, the accelerated electrons undergo betatron oscillations, generating a femtosecond pulse of synchrotron radiation, which has $\mathrm{keV}$ energy and lies within a narrow (50 $\mathrm{mrad})$ cone angle.
\end{abstract}

DOI: 10.1103/PhysRevLett.93.135005

$\mathrm{X}$-ray radiation has been, ever since its discovery over a century ago, one of the most effective tools used to explore the properties of matter for a broad range of scientific research. To continue delivering $\mathrm{x}$-ray beams with ever shorter wavelengths and higher brightnesses, successive generations of large-scale synchrotron facilities have been developed. Despite the remarkable progress that has been made by synchrotrons, there still exists a need for a light source that is capable of delivering $\mathrm{x}$ rays with femtosecond pulse duration, in order to provide the time resolution required to match the speed at which atomic-scale objects move $\left(10^{-13} \mathrm{~s} \hbar\right)$ [1-5]. Laser-driven light sources based on high-order harmonic generation have generated collimated beams of radiation with the required short pulse characteristics, but only with wavelengths as short as $10 \mathrm{~nm}$ (extreme ultra violet spectral range) [6]. Laser-driven $K \alpha$ x-ray sources [7-9] radiate subnanometer wavelength radiation, but not in collimated beams (instead, with isotropic angular distributions). In this paper, we discuss an alternative laser-driven light source capable of generating beams of ultrashort x-ray radiation, by means of wiggling an electron beam, as in a synchrotron, but with the laser plasma itself playing this role of both accelerator and wiggler.

In laser wakefield accelerators, the ponderomotive force of an intense femtosecond laser pulse generates, as it propagates in an underdense plasma, a large amplitude wakefield plasma wave. This wake can break, trap plasma electrons, and then its large electrostatic field can accelerate ultrashort pulse duration electron beams to high energies (a few hundreds of $\mathrm{MeV}$ ) on only a millimeter distance scale [10]. A particularly efficient mechanism for wakefield generation occurs in the parameter regime corresponding to ultrashort laser pulses ( $\sim 30 \mathrm{fs}$ ), called the forced laser wakefield regime, which has been studied
PACS numbers: 52.38.Ph, 52.25.Os, 52.38.-r, 52.50.Dg

experimentally [11] as well as with 3D particle-in-cell (PIC) simulations $[11,12]$. In this regime the wake has an electron-density depression right behind the laser pulse, leading to the formation of an ion column [13-15]. This charge displacement results in a strong radial electrostatic field. As the relativistic electrons propagate through these fields, they can undergo oscillations-called betatron oscillations - at a frequency given by $\omega_{b}=\omega_{p} / \sqrt{2 \gamma}$ (Figure 1). Here $\omega_{p}$ is the plasma frequency and $\gamma$ the relativistic factor of the electron. As in a conventional synchrotron, this oscillatory relativistic transverse motion produces a collimated x-ray beam $[13,14,16]$. But, because the wavelength of the wiggler can be much shorter in a laser-plasma interaction (micron-scale

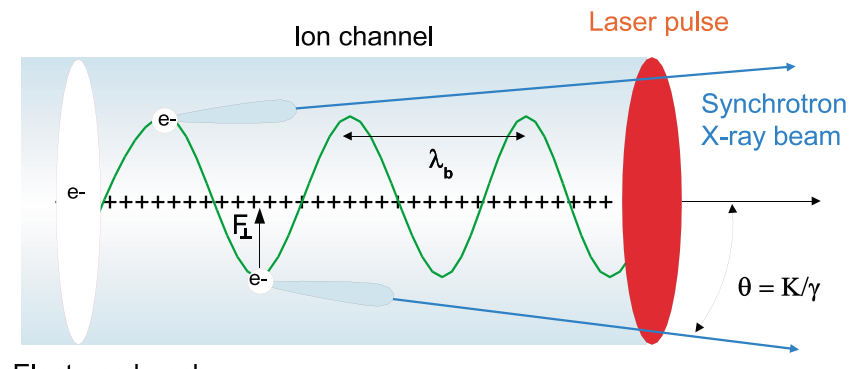

Electrons bunch

FIG. 1 (color online). Schematic representation of the synchrotron $\mathrm{x}$-ray source based on the betatron oscillation of a relativistic electron in a laser-produced ion channel. Electrons of the bunch experience the transverse electrostatic field of the channel; they make betatron oscillations and emit a femtosecond and collimated beam of synchrotron radiation in the $\mathrm{x}$ ray region. The figure shows a trajectory of an individual electron because the electrons of the bunch oscillate incoherently (all the phases are individual and uncorrelated). 
length) than in a synchrotron based on fixed magnets (centimeter-scale length), the distance required to produce a bright $\mathrm{x}$-ray beam is much shorter (on the scale of millimeters, rather than $10 \mathrm{~m}$ ); the required energy of the electron beam is also much lower $(\mathrm{MeV}$, rather than $\mathrm{GeV})$.

The characteristics of the radiation depend strongly on the amplitude $r_{0}$ of the betatron oscillation. For near-axis oscillations, the radiation is emitted at the fundamental wavelength given by $\lambda=\lambda_{b} /\left(2 \gamma^{2}\right)$, where $\lambda_{b}$ is the betatron wavelength. When the amplitude of the betatron oscillation becomes large (a few microns), high harmonics are radiated, and the resulting broadband spectrum is extended up to a critical energy $\hbar \omega_{c}[\mathrm{eV}]=5 \times$ $10^{-21} \gamma^{2} n_{e}\left[\mathrm{~cm}^{-3}\right] r_{0}[\mu \mathrm{m}]$ after which it drops exponentially. $n_{e}$ is the electron density of the plasma and $\omega_{c}$ is the critical frequency. The critical frequency is given by the relation $\omega_{c}=(3 / 2) \gamma^{2}\left|F_{\perp}\right| /(m c)$, where $F_{\perp}^{2}=F_{x}^{2}+$ $F_{y}^{2}$ is the square of the transverse force acting on relativistic electrons. The radiation is emitted in the forward direction within a cone of angle $K / \gamma$, where $K$ is the strength parameter of the plasma wiggler given by $K=$ $2 \pi\left(\gamma r_{0}\right) / \lambda_{b}=1.33 \times 10^{-10} \gamma^{0.5} n_{e}^{0.5}\left[\mathrm{~cm}^{-3}\right] r_{0}[\mu \mathrm{m}]$. The average number of photons with the mean energy $\hbar \omega_{c}$ emitted by one electron is given by $N_{\times}=5.6 \times$

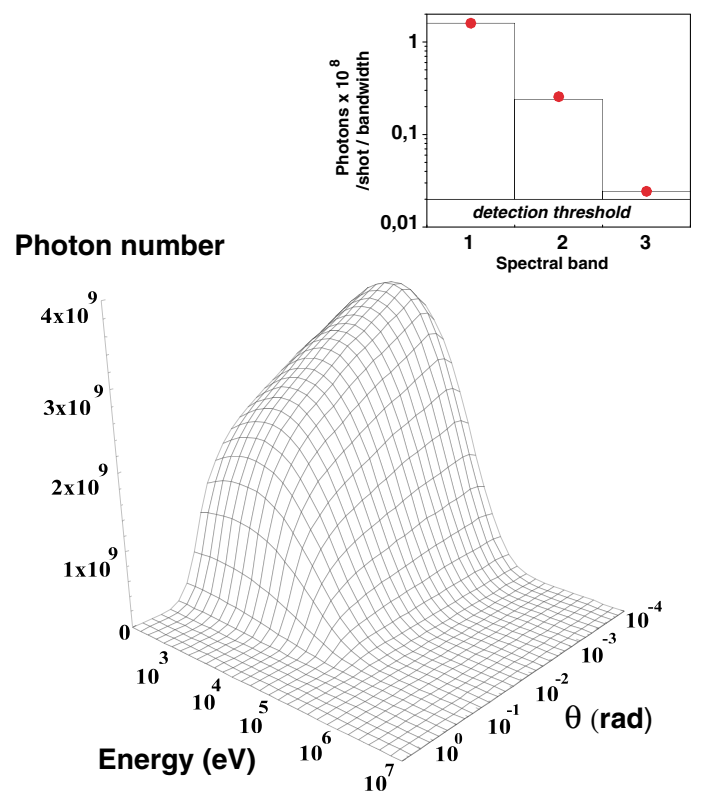

FIG. 2 (color online). The numerically predicted synchrotron spectrum from the plasma with density $n_{e}=1 \times 10^{19} \mathrm{~W} / \mathrm{cm}^{2}$ after $3 \mathrm{~mm}$ propagation distance. The distribution gives the number of x-ray photons emitted within $0.1 \%$ of the energy bandwidth $\left(\Delta \hbar \omega=10^{-3} \hbar \omega\right)$ per solid angle, $2 \pi \sin \theta d \theta$. The inset represents the $\mathrm{x}$-ray flux obtained experimentally, integrated over the beam divergence and in the spectral bandwidths determined by (1) $25 \mu \mathrm{m}$ Be filter $(1<E<10 \mathrm{keV})$, (2) $25 \mu \mathrm{m} \mathrm{Be} \mathrm{filter}+40 \mu \mathrm{m} \mathrm{Al} \mathrm{filter}(4<E<10 \mathrm{keV})$, and (3) $25 \mu \mathrm{m} \mathrm{Be}$ filter $+25 \mu \mathrm{m} \mathrm{Cu}$ filter $(6<E<10 \mathrm{keV})$.
$10^{-3} N_{0} K$, where $N_{0}$ is the number of betatron oscillations accomplished by the electron.

To get a better description of the x-ray properties, we have simulated the laser-plasma interaction in this highlaser-intensity regime with a 3D PIC code [17], which has been modified to properly model the synchrotron emission. In the code, we suppose that at any given moment of time, the relativistic electron emits, along its momentum direction, a radiation spectrum defined by the universal
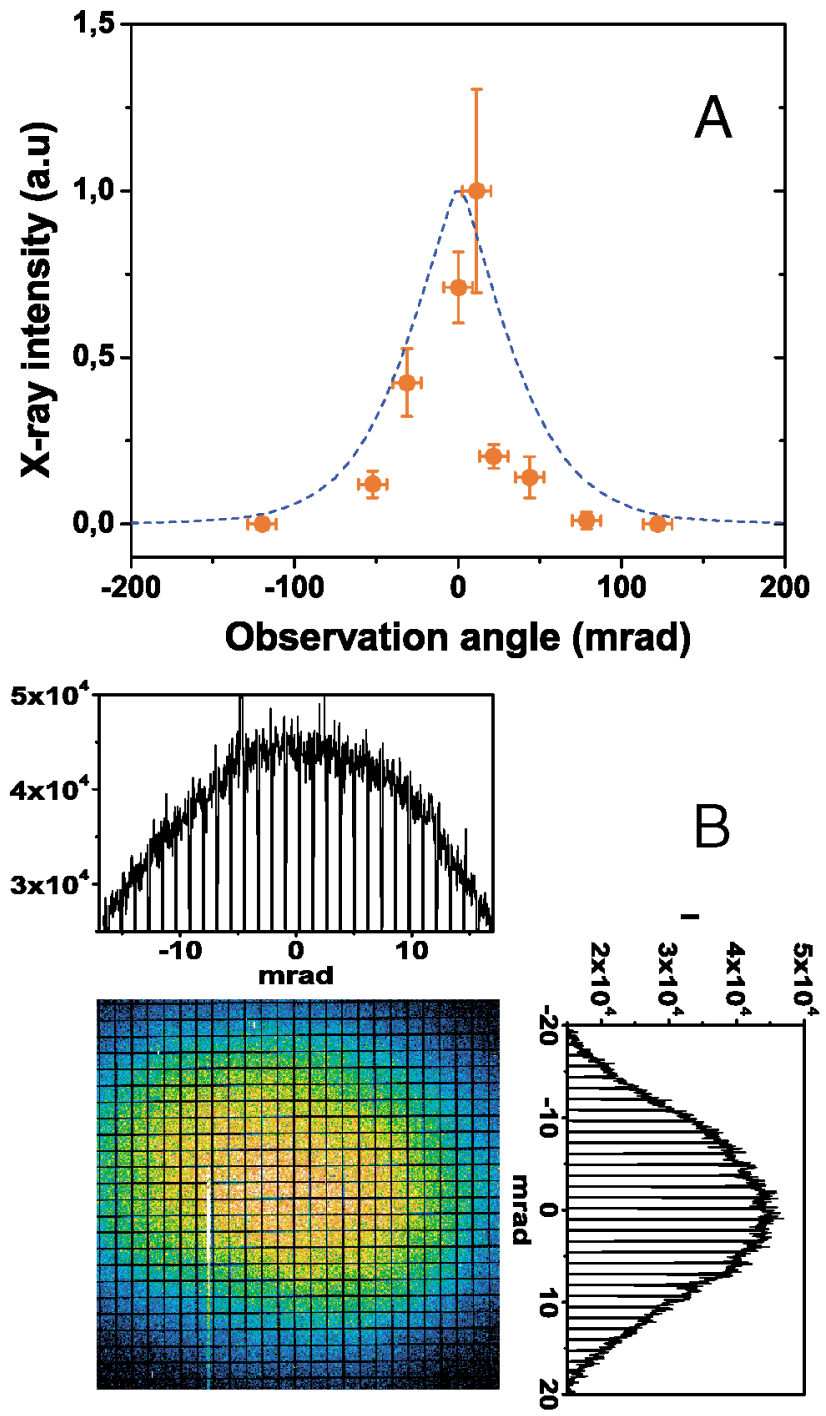

FIG. 3 (color). Angular distribution of the radiation for x-ray energies beyond $1 \mathrm{keV}$. (a) The measurement is made for $n_{e}=$ $10^{19} \mathrm{~cm}^{-3}$ in the horizontal plane. Each point corresponds to an average value over ten shots. The dotted line represents the result obtained from the 3D PIC simulation. (b) Spatial profile of the $\mathrm{x}$-ray beam at $n_{e}=8 \times 10^{18} \mathrm{~cm}^{-3}$. The corresponding lineout graphics, where the shadow of a nickel grid placed in the beam appears, provides the radiation source size in the transverse directions. We obtain a source size of $20 \mu \mathrm{m} \times$ $20 \mu \mathrm{m}$. 
function $S\left(\omega / \omega_{c}\right)$ [18]. We follow the trajectories of each electron and calculate the emission during the interaction. The recoil on the electron [18] exerted by the emitted radiation is included into the equations of the electron motion. In the calculation we have used a Gaussian laser pulse $a(t, r)=a_{0} \exp \left(-r_{\perp}^{2} / r_{L}^{2}-t^{2} / T_{L}^{2}\right)$ with a wavelength $\lambda=0.820 \mu \mathrm{m}$, where $a=e A / m c^{2}$ is the normalized vector potential, initially $a_{0}=1.2, r_{L}=18 \mu \mathrm{m}$, and $T_{L}=30 \mathrm{fs}$. For these parameters, the self-focusing is moderate because the laser pulse duration is shorter than the plasma period. It leads to an intensity increase by a factor of 2 to 3 . The maximum $\mathrm{x}$-ray emission is observed at $n_{e}=1 \times 10^{19} \mathrm{~cm}^{-3}$. At this density, the wake amplitude maximizes because the laser pulse duration resonantly fits the relativistically corrected plasma wave period $T_{p}=2 \pi / \omega_{p}$, where $\omega_{p}^{2}=4 \pi n_{e} e^{2} /(\gamma m)$. The x-ray spectrum obtained numerically for the optimal plasma density $n_{e}=1 \times 10^{19} \mathrm{~cm}^{-3}$ and for a laser propagation distance of $3 \mathrm{~mm}$ is shown in Fig. 2. It gives the number of x-ray photons emitted within $0.1 \%$ of the energy bandwidth $\left(\Delta \hbar \omega=10^{-3} \hbar \omega\right)$ per solid angle $(2 \pi \sin \theta d \theta)$. The energy and the angle $\theta$ axes are logarithmically scaled. The maximum of the $\mathrm{x}$-ray emission is located in the energy range $1-10 \mathrm{keV}$, and the divergence of the $\mathrm{x}$-ray beam is about $50 \mathrm{mrad}$ (FWHM).

The experiment was performed at the Laboratoire d'Optique Appliquée using a titanium-doped sapphire (Ti:sapphire) laser operating at $10 \mathrm{~Hz}$ with a wavelength $\lambda_{0}$ of $820 \mathrm{~nm}$ in chirped-pulse amplification mode [19]. The laser delivered energies up to $1 \mathrm{~J}$ on target in $30 \mathrm{fs}$, with a linear horizontal polarization. The laser beam was focused with an $f / 18$ off-axis parabolic mirror onto the edge of a supersonic helium gas jet (diameter $3 \mathrm{~mm}$ ). The laser distribution in the focal plane was Gaussian with a waist $w_{0}$ of $18 \mu \mathrm{m}$ containing $50 \%$ of the total laser energy. This produces vacuum-focused intensities $I_{L}$ on the order of $3 \times 10^{18} \mathrm{~W} / \mathrm{cm}^{2}$, for which the corresponding normalized vector potential $a_{0}$ is 1.2. In this experiment we measured, by using a magnetic spectrometer, an electron beam which has a temperature of $20 \mathrm{MeV}$. Finally, the Thomson imaging of the plasma shows direct evidence of self-focusing over a millimeter. We have measured the $\mathrm{x}$-ray radiation produced in the plasma using a cooled $\mathrm{x}$-ray CCD camera placed directly on the laser axis without any focusing x-ray optic (Fig. 3). For all the measurement, a $25 \mu \mathrm{m}$ beryllium filter is kept in front of the CCD camera to block any radiation below $0.8 \mathrm{keV}$. Permanent magnets $(0.3 \mathrm{~T}$ along $20 \mathrm{~cm})$ are inserted between the plasma and the x-ray CCD to deviate off axis the accelerated charged particles. Even if the most energetic electrons are still recorded onto the detector, they are significantly out of the x-ray axis. Finally, we estimated the spectrum of the radiation by using an additional set of aluminum, nickel, and copper filters.
The spectral and angular features of the $\mathrm{x}$-ray emission as well as its dependency on the electronic density of the plasma have been characterized experimentally. The experimental results are found to be in good agreement with the numerical simulations and the analytical estimations describing the radiation emitted by the trapped electrons undergoing betatron oscillations in the ion channel. At first, $\mathrm{x}$ rays were detected from 1 to $6 \mathrm{keV}$ (Figs. 2 and 3). The back-illuminated CCD, which is not sensitive to energies above $10 \mathrm{keV}$, did not allow a full spectral characterization of the radiation. The inset of Fig. 2 represents the spectral distribution obtained by using $\mathrm{Be}, \mathrm{Al}, \mathrm{Cu}$, and Ni filters. The total number of photons integrated over the bandwidths of the filters is more than $10^{8}$ photons, per shot and integrated over all angles, which is in close agreement with the result expected from the simulation also shown in Fig. 2. Second, the radiation is found to be collimated in a narrow cone centered on the laser axis (Fig. 3). For plasma parameters at which the x-ray intensity is maximum, the spatial distribution is larger than the size of the CCD area (using our experimental setup) and is obtained by rotating the $\mathrm{X}$-ray CCD around the gas jet. The $\mathrm{x}$-ray beam divergence, averaged over more than ten shots, is found to be $\Delta \theta=50 \pm 20 \mathrm{mrad}$ at FWHM. More collimated $\mathrm{x}$-ray beams have been observed at slightly lower electron density where the $\mathrm{x}$-ray signal is weaker. As shown from the beam profile of Fig. 3, the beam divergence can reach $20 \mathrm{mrad}$ (FWHM) in that case. A third striking feature of the observed $x$-ray emission is its intensity as a function of the electron density of the plasma. We found that the radiative process is more efficient at the plasma density $n_{e-\max }=1.1 \times 10^{19} \mathrm{~cm}^{-3}$ at which the x-ray intensity is sharply peaked (Fig. 4). The PIC numerical simulations clearly reproduce this experimental behavior (Fig. 4): a sharp increase of the

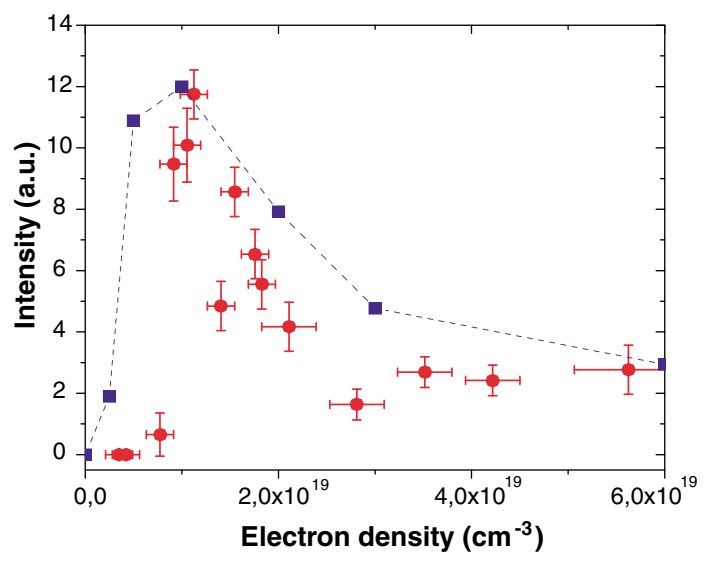

FIG. 4 (color online). X-ray signal as a function of the plasma electron density for $\mathrm{x}$-ray energies beyond $1 \mathrm{keV}$. Each point corresponds to an average value over ten shots. The dotted line corresponds to the result obtained using a 3D PIC simulation. 
$\mathrm{x}$-ray intensity followed by a smoother decrease of the signal. For $n_{e}<n_{e-\max }$, the $\mathrm{x}$-ray signal vanishes because self-focussing of the laser beam does not occur and the plasma wave generation is inefficient. This is confirmed in the experiment for which no electrons were detected by our spectrometer. At larger densities, the x-ray signal drops down and a plateau is reached. For these experimental conditions, the laser must first be modulated to create high amplitude plasma waves [20] and much higher laser intensity is needed.

Other radiative processes could potentially produce a collimated beam of $\mathrm{x}$ rays in the $\mathrm{keV}$ energy range. Relativistic bremsstrahlung radiation is emitted by the electrons undergoing collisions with the ionic background. However, its intensity is too low under our experimental conditions to explain the results. Nonlinear Thomson scattering radiation, emitted by the electrons of the plasma oscillating in the intense laser field, has also been proposed as a source of femtosecond $\mathrm{x}$ rays [21] and results in the $100 \mathrm{eV}$ range (for $a_{0} \sim 6$ ) [22] have been already obtained. However, much higher laser intensity (at least $a_{0} \sim 10$ ), compared to our experiment, is needed to produce $\mathrm{keV}$ radiation.

The brightness of produced $\mathrm{x}$-ray radiation is estimated from the pulse duration and the size of the $\mathrm{x}$-ray source. The temporal pulse width is fully determined by the temporal profile of the electron bunch, which is close to that of the laser (30 fs) $[10,11]$. The shadow of a wire of a mesh positioned in the $\mathrm{x}$-ray beam was used as a "knife edge" to perform transverse source size measurements. We obtained a source of $20 \times 20 \mu \mathrm{m}$, which leads to an average brightness of $5 \times 10^{6} \mathrm{ph} / \mathrm{s} / \mathrm{mm}^{2} / \mathrm{mrad}^{2} / 0.1 \%$ bandwidth (BW) and a peak spectral brightness is $2 \times$ $10^{22} \mathrm{ph} / \mathrm{s} / \mathrm{mm}^{2} / \mathrm{mrad}^{2} / 0.1 \% \mathrm{BW}$. The $\mathrm{x}$-ray flux could be further enhanced by increasing the laser energy (we found a quadratic dependency of the x-ray flux in the experiment) as well as the propagation length of the laser (proportional to $N_{0}$ [23]). The x-ray energy can also be extended to harder spectral range by increasing the strength parameter $K$ of the plasma wiggler. In the near future, the anticipated higher laser energy [24] as well as the more energetic electrons will improve the capabilities of the source.

Our results demonstrate that ultrashort-duration pulses of $\mathrm{x}$-ray beams can be produced by the synchrotron radiation mechanism in a compact device, which is based on a high-field femtosecond laser. $5 \times$ $10^{6}$ photons/pulse/s/0.1\% BW are produced and the $\mathrm{x}$ rays are perfectly synchronized with the laser system, enabling visible pump/x-ray probe experiments with insignificant time-jitter. Laue diffraction and absorption $\mathrm{x}$-ray studies can be achieved, which could significantly extend the first $\mathrm{x}$-ray diffraction studies already demonstrated in ultrafast $x$-ray science [25-28].
This work was supported by the European Community under contracts no. HPRI-CT-1999-00086, HPRI-CT2000-40016, and HPRI-CT-1999-50004 (FAMTO project). K.T.P. was supported by the NSF FOCUS Center, and D. U. and R.S. by the Chemical Sciences, Geosciences, and Biosciences Divisions of the Office of Science, U.S. DOE.

*Corresponding author.

Electronic adress: kim.taphuoc@ensta.fr

[1] Ultrafast Phenomena proceedings, Springer Ser. Chem. Phys. Vol. 55, 60, 62, 63, and 66(Springer, Berlin, 19922002).

[2] A. H. Zewail, J. Phys. Chem. A 104, 5660 (2000).

[3] N. Bloembergen et al., Rev. Mod. Phys. 71, S283 (1999).

[4] A. Rousse et al., Rev. Mod. Phys. 73, 17 (2001).

[5] R. F. Service, Science 298, 1356 (2002).

[6] A. Tarasevitch et al., Phys. Rev. A 62, 023816 (2000); A. Th. Brabec and F. Krausz, Rev. Mod. Phys. 72, 545 (2000).

[7] A. Rousse et al., Phys. Rev. E 50, 2200 (1994).

[8] M. Murnane et al., Science 251, 531 (1991).

[9] J. C. Kieffer et al., Phys. Fluids B 5, 2676 (1993).

[10] D. Umstadter, J. Phys. D 36, R151 (2003).

[11] V. Malka et al., Science 298, 1596 (2002).

[12] A. Pukhov and J. Meyer ter Vehn, Appl. Phys. B 74, 355 (2002).

[13] I. Kostyukov, A. Pukhov, and S. Kiselev, Phys. Plasmas 10, 4818 (2003).

[14] E. Esarey et al., Phys. Rev. E 65, 056505 (2002).

[15] D. H. Whittum, Phys. Fluids B 4, 730 (1992).

[16] The synchrotron radiation emitted from electrons undergoing betatron motion has been studied numerically and theoretically [5,6], and observed at the Stanford linear accelerator where $5 \mathrm{ps} x$-ray pulses were produced using a $28.5 \mathrm{GeV}$ energy electron bunch that was sent into a $1.4 \mathrm{~m}$ long plasma. [See S. Wang et al., Phys. Rev. Lett. 88, 135004-1 (2002), and C. Joshi et al., Phys. Plasmas 9, 1845 ( 2002)].

[17] Virtual Laser-Plasma Laboratory PIC code. It is discussed in $[6,7]$

[18] J. D. Jackson, Classical Electrodynamics (Wiley, New York 1975). Here, we used $S(x)=x \int_{x}^{\infty} K_{5 / 3}(\xi) d \xi$.

[19] M. Pittman et al., Appl. Phys. B 74, 529 (2002).

[20] V. Malka et al., Phys. Plasmas 8, 2605 (2001).

[21] Y.Y. Lau, F. He, and D. Umstadter, Phys. Plasmas 10, 2155 (2003).

[22] K. Ta Phuoc et al., Phys. Rev. Lett. 91, 195001 (2003).

[23] The simulation shows that the accelerated electrons experience five betatron oscillations in our parameter regime.

[24] M. Aoyama, Opt. Lett. 28, 1594 (2003).

[25] C. Rischel et al., Nature (London) 390, 490 (1997).

[26] C. Siders et al., Science 286, 1340 (1999).

[27] A. Rousse et al., Nature (London) 410, 65 (2001).

[28] K. Sokolovski-Tinten et al., Nature (London) 422, 287 (2003). 